\title{
DNA methylation is a key mechanism for maintaining monoallelic expression on autosomes
}

\author{
Saumya Gupta ${ }^{1,2,3 *}$, Denis L. Lafontaine ${ }^{1 *}$, Sebastien Vigneau ${ }^{1,2}$, Svetlana Vinogradova1 ${ }^{1}$, Asia Mendelevich ${ }^{1,4}$, Kyomi J. \\ Igarashi ${ }^{1}$, Andrew Bortvin ${ }^{1}$, Clara F. Alves-Pereira ${ }^{1,2,3}$, Kendell Clement ${ }^{3,5}$, Luca Pinello ${ }^{3,5}$, Andreas Gnirke ${ }^{3}$, Henry Long ${ }^{6}$, \\ Alexander Gusev ${ }^{7}$, Anwesha Nag ${ }^{1}$, Alexander A. Gimelbrant ${ }^{1,2,3 * *}$
}

\begin{abstract}
${ }^{1}$ Dana-Farber Cancer Institute, Department of Cancer Biology and Center of Cancer Systems Biology, Boston, MA; ${ }^{2}$ Harvard Medical School, Department of Genetics, Boston, MA; ${ }^{3}$ Broad Institute of MIT and Harvard, Cambridge, MA; ${ }^{4}$ Skolkovo Institute of Science and Technology, Moscow, Russia;

${ }^{5}$ Molecular Pathology Unit and Center for Cancer Research, Massachusetts General Hospital, Charlestown, MA; ${ }^{6}$ Dana-Farber Cancer Institute, Center for Functional Cancer Epigenetics, Boston, MA; ${ }^{7}$ Dana-Farber Cancer Institute, Department of Medical Oncology and Population Sciences, Boston, MA *Equal contribution; ** Lead contact: gimelbrant@mail.dfci.harvard.edu (AAG)
\end{abstract}

\section{Abstract}

In diploid cells, maternal and paternal copies of genes usually have similar transcriptional activity. Mammalian allele-specific epigenetic mechanisms such as $\mathrm{X}$-chromosome inactivation $(\mathrm{XCl})$ and imprinting were historically viewed as rare exceptions to this rule. The discovery of mitotically stable monoallelic autosomal expression (MAE) a decade ago revealed an additional allele-specific mode regulating thousands of mammalian genes. However, despite its prevalence, the mechanistic basis of MAE remains unknown. To uncover the mechanism of MAE maintenance, we devised a small-molecule screen for reactivation of silenced alleles across multiple loci using targeted RNA sequencing. Contrary to previous reports, we identified DNA methylation as a key mechanism of MAE mitotic maintenance. In contrast with the binary choice of the active allele in XCI, stringent transcriptome-wide analysis revealed MAE as a regulatory mode with tunable control of allele-specific expression, dependent on the extent of DNA methylation. In a subset of MAE genes, allelic imbalance was insensitive to changes in DNA methylation, implicating additional mechanisms in MAE maintenance in these loci. Our findings identify a key mechanism of MAE maintenance, reveal tunability of this mode of gene regulation, and provide the essential platform for probing the biological role of MAE in development and disease.

\section{INTRODUCTION}

In mammalian cells, the maternal and paternal gene copies tend to make an equal contribution to transcription (1). However, several allele-specific modes of gene regulation provide important exceptions. One such mode is genomic imprinting, where the allelic choice is determined by the parent of origin in about 200 mammalian genes (2). Another is X-chromosome inactivation $(\mathrm{XCl})$, which randomly silences one of the two copies of the $X$ chromosome in females (3), affecting over 800 X-linked genes. Additionally, olfactory sensory neurons express one allele of one out of $\sim 1000$ olfactory receptor genes (4).

The discovery of widespread monoallelic autosomal expression (MAE) greatly expanded our view of allelespecific gene regulation (5). Like $\mathrm{XCl}, \mathrm{MAE}$ involves a random choice of the active allele during development, resulting in an epigenetic mosaic (6). Also like $\mathrm{XCl}$, the allelic choice in MAE genes is mitotically stable; however,
MAE genes can be expressed from both alleles in a subset of clonal lineages (Fig.1A). MAE had been observed in clonal populations of every cell type assessed (5, 7-13) and most MAE genes are highly cell-type specific (14). Cumulatively across cell types, an estimated 4000 human genes are subject to MAE (15), including genes implicated in cancer, neurodevelopmental disorders, and other diseases.

The biological role of widespread MAE is presently unclear, though multiple lines of evidence imply it has a significant impact on organismal function. MAE has been shown to result in dramatic functional differences between otherwise similar cells; for example, the function of $B$ cells in mice heterozygous for TIr4 depends on which allele is active in a given cell (16). Evolutionary and populationgenetic analyses indicate conservation of the MAE status between human and mouse $(13,17)$, and selective advantage for individuals heterozygous for MAE genes $(18,19)$. The prevalence of cell surface molecules among 
proteins encoded by MAE genes prompted the hypothesis that MAE leads to increased variation in responses to extrinsic signals between otherwise similar cells (18).

Lack of knowledge about the underlying mechanism of MAE has severely limited research on MAE function. At present, no perturbation is known to affect the maintenance of allele-specific silencing in any MAE locus (20). MAE status correlates with histone modifications and DNA methylation in the gene body and putative regulatory sequences $(8,9,12,17,21)$. However, inhibiting the DNA methyltransferases was reported to not affect the allelic imbalance in any of the tested MAE genes $(8,9)$, arguing against a mechanistic role of DNA methylation in MAE (20).

To understand the mechanistic basis of MAE, we devised a novel strategy for screening by targeted RNA sequencing and performed a small molecule screen for perturbations that affect allelic imbalance in expression in any of the 23 targeted genes across the mouse genome. We found that inhibition of methyltransferase Dnmt1-

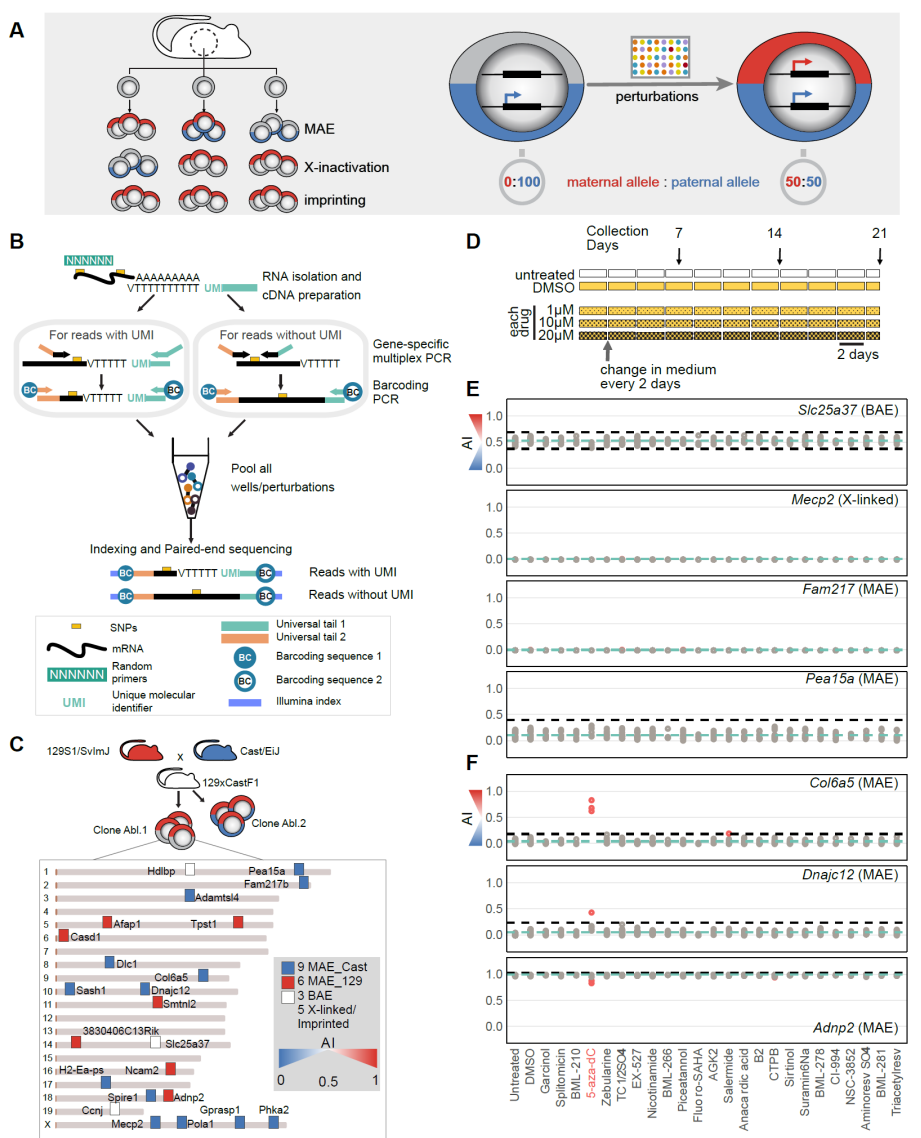

dependent DNA methylation reactivated silenced alleles in many MAE loci, showing that DNA methylation plays a major role in the MAE mitotic maintenance. At the same time, many MAE loci showed no significant changes in allele-specific expression upon DNA demethylation, suggesting that MAE is mechanistically heterogeneous. Application of a highly stringent statistical approach to transcriptome-wide differential analysis of allele-specific expression (22) revealed the existence of multiple mitotically stable states of allelic imbalance, which correlated with the extent of DNA methylation. We conclude that DNA methylation acts as a fine-tuning mechanism for MAE loci, controlling allele-specific transcription as a rheostat, as opposed to an on-off switch.

\section{RESULTS}

\section{Screening-by-sequencing approach for sensitive detection of allele-specific expression}

To screen for reactivation of a silenced allele, we looked for shifts in allelic imbalance (Al, the fraction of one

Figure 1. Perturbations that reactivate silenced alleles of genes with monoallelic expression identified using screening-by-sequencing.

(A) Left: Different epigenetic modes of monoallelic expression. Note that while imprinting is uniform across cells, $X$ chromosome inactivation and autosomal MAE result in epigenetic clonal mosaicism. Right: logic of the screen illustrated with a single locus in a clone with a completely silenced maternal allele. (B) Outline of Screen-seq methodology. Top to bottom: Cells are lysed in-plate, and in each well, RNA is isolated using SPRI beads. Two types of SNPs between parental genomes for the readout genes are targeted: those close to the poly-A tail enabling use of the UMI (left) and the rest that were targeted with two genespecific primers with universal tails (right). Well-encoding is performed using primers targeting common adapters coupled with barcodes (BC1 and BC2). Then, all wells are pooled, Illumina sequencing adapters are added, and the pooled library is sequenced. See also Suppl. Fig.S1 and S2. (C) 23 genes assayed in Screen-seq and their distribution in the mouse genome. Allelic imbalance (AI) of target genes in Abl. 1 clone is reflected by the marker color. Centromeres (brown) on the left. (D) Drug treatment setup. Each of the tested 48 drugs were used in three concentrations: $1 \mu \mathrm{M}, 10$ $\mu \mathrm{M}$ and $20 \mu \mathrm{M}$ in 1\% DMSO. Fresh media and drugs were replaced every 2 days. Cells were collected on day 7, 14 and 21 and processed for Screen-seq. (E, F) Screen-seq results for a representative set of readout genes. All time and concentration points for a single drug shown in the same column. Each point shows allelic imbalance in one condition $(\mathrm{Al}=[129$ counts $] /[129+$ Cast counts]). Blue line: mean Al for a gene across all conditions; black dashed lines: [Q1-3×IQR] and [Q1+3×IQR] (inter-quartile range); red points: outlier $\mathrm{Al}$ values (hits). (E) - genes showing no Al change in any condition. (F) - genes with significant changes in some conditions. Screen-seq results for all readout genes are in Suppl. Fig.S3. 
allele over the total allelic counts; Fig.1A, right) upon drug treatment. In order to increase the likelihood of detecting Al shifts among genes with potentially different regulation, our screening approach would ideally combine the ability to assess multiple readout genes, sensitivity to $\mathrm{Al}$ changes, and the throughput to process multiple samples after exposure to an array of perturbations.

We designed a screening-by-sequencing strategy, Screen-seq, to satisfy all of these requirements. In cells with heterozygous genomes, allele-specific expression can be assessed without the need for any engineered reporters and by relying on the detection of single nucleotide polymorphisms (SNPs). Precision and sensitivity of the Al measurement in RNA sequencing critically depend on the depth of SNP coverage (22). Sequencing of SNP-containing amplicons from multiplexed RT-PCR as the readout allows for very deep coverage and thus a highly precise Al measurement.

The experimental flow of Screen-seq is outlined in Fig.1B. Cells were grown and lysed in 96-well plates; RNA isolated using magnetic beads, and cDNA synthesized with a mix of random primers and oligo-dT primers with Unique Molecular Identifiers [UMls, $(23,24)]$. This mix allowed targeting of two types of SNPs in the next step, multiplex PCR: SNPs close to the 3'-end enable the use of oligo-dT-UMls followed with a gene-specific primer, while other SNPs were targeted with two genespecific primers in random-primed cDNA. Next, plate- and well-encoding barcodes were added using PCR. The reactions from all the wells were pooled, Illumina adaptors added, and the pooled library was sequenced. Finally, SNP counts were assigned to specific genes, and barcodes to specific plates and wells with a specific perturbation.

To allow analysis of MAE genes, which show different Al in different clones, we performed our screen in a monoclonal line of pro-B cells (Abl.1). We have previously characterized allele-specific expression in several such clones, including Abl.1 and other clones used in this study $(13,14)$. These cells were derived from a female 129S1/SvImJ $\times$ Cast/EiJ F1 mouse, then immortalized using the Abelson murine leukemia virus (25) and cloned through single-cell sorting. In this mouse cross, the median distance between SNPs in the non-repetitive genome is $\sim 80$ bp and almost all cDNAs have at least one informative SNP.

For readout, we selected 27 SNPs in 23 target genes across the genome, including 15 clone-specific MAE genes as well as three biallelic, one imprinted and four Xinactivated loci (Fig.1C, Suppl. Table S1). The selected MAE genes showed $\mathrm{Al}>0.9$ or $\mathrm{Al}<0.1$ in the Abl.1 clone $(\mathrm{Al}=[129$ counts]/[129 + Cast counts]), while showing opposite bias or biallelic expression in another clone, Abl.2 $(13,14)$. Targeted MAE genes spanned a range of expression levels and extent of allelic bias in the screening clone, Abl.1; some showed complete silencing of one allele (such as Afap1, Al = 1.0), while others showed strong but incomplete bias (such as Dlc1, Al = $0.1)$.

We first tested that these assays were able to detect changes in Al. Since no perturbations are known that can change $\mathrm{Al}$ in any locus, much less in all targeted loci, for the control experiments we titrated known mixes of genomic DNA from liver tissue of the parental mouse strains, 129S1/SvImJ and Cast/EiJ. Expected and measured Al were highly concordant $\left(R^{2} \geq 0.99\right)$ at $>1000$ reads/SNP (Suppl. Fig.S1). We also compared Al sensitivity for UMI and non-UMI assays, by designing both types of assays for a subset of genes where the position of SNPs allowed that. For this, we used mixes prepared from total RNA from the spleens of the mice of the parental mouse strains. Al measurements were highly concordant between the UMI and non-UMI assays $\left(R^{2} \geq\right.$ 0.97, Suppl. Fig.S2).

Based on these pilot experiments, we concluded that Screen-seq can be used for sensitive detection of $\mathrm{Al}$ changes in the targeted loci.

\section{Identification of perturbations that affect allele- specific gene expression}

Clone-specific MAE has been associated with specific chromatin signatures, i.e., combinations of histone modifications in human and mouse cells $(8,12,14)$, suggesting that chromatin modifying mechanisms might be involved in MAE maintenance. We thus assessed the impact on $\mathrm{Al}$ in the targeted loci of treatment with a set of 43 small molecules with known effects on the activity of the enzymes involved in the deposition and removal of methylation and acetylation marks on histones and DNA (Suppl. Table S3). Abl.1 cells in 96-well plates were exposed for 21 days to individual drugs in regular growth conditions (Fig.1D). Each drug was applied in three final concentrations ( $1 \mu \mathrm{M}, 10 \mu \mathrm{M}$ and $20 \mu \mathrm{M}$ in $1 \%$ DMSO). Controls were untreated cells and cells with only solvent (1\% DMSO) added. Fresh media (with or without drugs, as appropriate) was replaced every two days. On days 7 , 14 , and 21 , aliquots of cells were removed for analysis. 
For 19 of the 43 drugs, no live cells were evident after six days, at any drug concentration. Each cell collection thus involved only 72 wells with treated cells (24 remaining drugs at three concentrations) and 24 wells with controls (12 untreated and 12 vehicle-treated cells). Taken together, in this Screen-seq experiment we assessed 7,776 experimental points (allele-specific measurements of 27 SNPs in 23 genes $\times 96$ wells $\times 3$ time points).

With a targeted RNA-seq library, only a very moderate amount of sequencing was needed to reach the coverage depth required for sensitive allele-specific analysis. At 1,000 reads per experimental point, fewer than $10 \times 10^{6}$ sequenced fragments were needed for the entire screen.

As potential hits, we identified conditions resulting in outlier Al values (Fig.1E,F; see Methods for details). Al measurements were highly uniform for some genes (e.g. Fam27b or Mecp2) across drug concentrations and time points, while there was more variation in other genes (e.g., Pea15a or Col6a5). To allow for variation in assay sensitivity, each readout gene was analyzed independently of the rest. Outliers were identified using highly stringent criteria (see Methods).

As expected for stably maintained allele-specific expression, in the untreated cells there were no outliers for any of the readout genes. The most pronounced outliers (red in Fig.1F) were observed for 3 MAE readout genes in the presence of 5-aza-2'-deoxycytidine (5-aza$\mathrm{dC}$ ). There were also significant $\mathrm{Al}$ shifts in single reporter genes after exposure to histone deacetylase modulators Salermide and BML-278 (complete Screen-seq results are in Suppl. Fig.S3 and Suppl. Table S4). The magnitude of the observed shifts varied between genes and conditions, including drug concentration and exposure times. The most striking example is a shift in Col6a5 gene from baseline $\mathrm{Al} \approx 0.1$ baseline in the control to $\mathrm{Al} \approx 0.8$ after 7 days in the presence of $1 \mu \mathrm{M} 5$-aza-dC (Fig.1F). More subtle, significant shifts were observed for the MAE genes Adnp2 (from $\mathrm{Al}=1.0$ to $\mathrm{Al}=0.8$ ) and Dnajc12 (Al $\approx 0.1$ to $A l \approx 0.2$ ). Notably, in other tested genes, no Al shift was observed in 5-aza-dC (Suppl. Fig.S3). We focused on characterizing the strongest primary hit, 5-aza-dC.

\section{5-aza-dC affects allele-specific expression of autosomal MAE genes via DNA demethylation}

To validate the candidate hit 5-aza-dC, a classic DNA demethylation agent (26), we performed several sets of experiments. First, we took advantage of the fact that the
Screen-seq protocol leaves enough RNA and cDNA for re-testing. We measured $A l$ in the same samples using an orthogonal method, droplet digital PCR (ddPCR, a highly sensitive approach to measuring allelic frequencies (27)). In addition to using a different readout method, we assessed different SNPs than those used for Screen-seq for the same genes. Using cDNA from cells treated with 1,10 and $20 \mu \mathrm{M}$ of 5 -aza-dC for 7 days, we performed ddPCR to assess reactivation of the silenced maternal allele of the Col6a5 and Dnajc12 genes. Confirming the results from Screen-seq, ddPCR measurements showed a similarly striking shift in Col6a5 Al from a paternal bias to maternal bias ( $A \mathrm{l}=0.1$ to 0.8 ) after 7 days in $1 \mu \mathrm{M} \mathrm{5-}$ aza-dC (Fig.2A,B). Also confirming the Screen-seq results, Al for Dnajc12 gene showed relaxation towards a more biallelic expression, with Al shifting from 0 to 0.1 in $1 \mu \mathrm{M} 5$-aza-dC and to 0.3 in $20 \mu \mathrm{M} 5$-aza-dC in 7 days (Fig.2B).

In biological replicate experiments, the Abl.1 clonal cells were exposed to a range of concentrations of 5-aza$\mathrm{dC}$ for varying times. Using ddPCR, we observed that the maternal allele of Col6a5 was reactivated in a dose- and time-dependent manner (Fig.2C,D). Al shifts for Dnajc12 and Adnp2 were also concordant with those observed in Screen-seq (Fig.2D and Suppl. Fig.S4). Taken together, these observations show that 5-aza-dC causes a shift in allelic imbalance in a subset of MAE genes.

A closely related compound, 5-aza-cytidine (5-aza-C), is also a well-known demethylating agent, although less potent and toxic than 5-aza-dC (28). Since 5-aza-C was not one of the perturbagens tested in our screening, we assessed whether it had a similar effect as 5-aza-dC on Al changes. Within 2 days of treatment with $10 \mu \mathrm{M} 5$-aza$\mathrm{C}$, the $\mathrm{Al}$ of Col6a5 shifted from 0 to 0.2 , and to 0.6 after 5 days in $2 \mu \mathrm{M}$ 5-aza-C (Suppl. Fig.S5). Another MAE readout gene, Dnajc12, showed a shift in Al from 0 to 0.1 within 2 days in $2 \mu \mathrm{M} 5$-aza-C. This further supports the role of DNA methylation in MAE maintenance.

5-aza compounds at high concentrations are cytotoxic and cause cell cycle arrest (29). We asked whether shifts in $\mathrm{Al}$ in the target genes might be due to nonspecific cytotoxicity. In the presence of $2 \%$ DMSO, higher than the $1 \%$ concentration used as a drug solvent, the Abl. 1 clonal cells viability was reduced to $34 \%$ after 2 days, similar to their viability after 5 days in $2.5 \mu \mathrm{M}$ 5-aza-dC (Suppl. Fig.S6). In contrast to the Al shifts in the presence of 5aza-dC and 5-aza-C (Fig.2D and Suppl. Fig.S5), no changes in Al were observed for the MAE readout genes, Col6a5 and Dnajc12, in 2\% DMSO (Suppl. Fig.S7), 
A
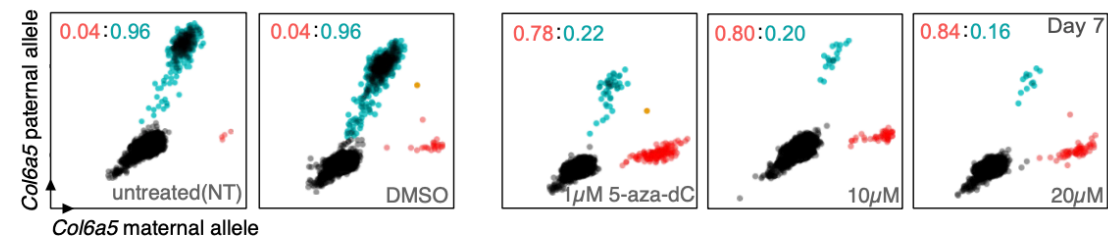

$\mathrm{B}$
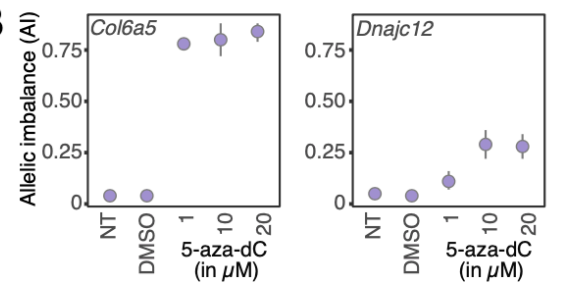

C
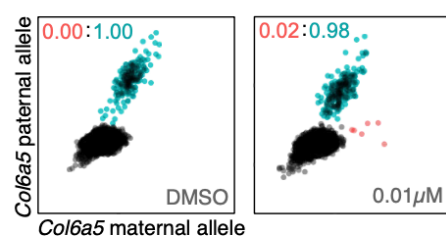

$0.01 \mu \mathrm{M}$
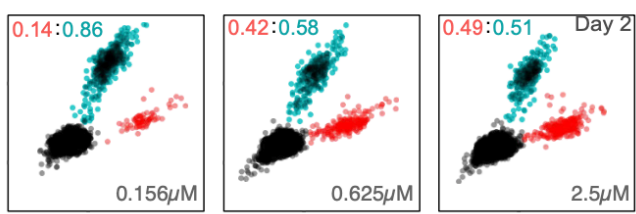

$\mathrm{D}$
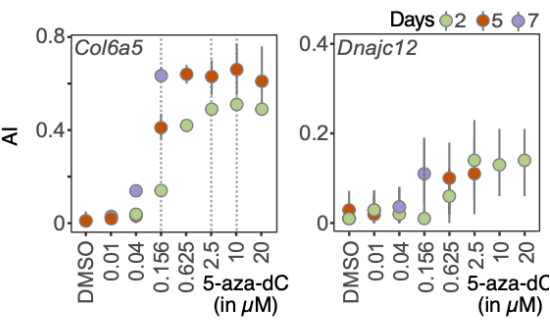

E

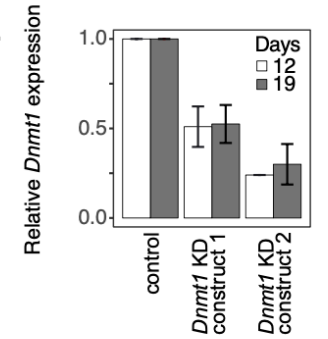

$\mathrm{F}$

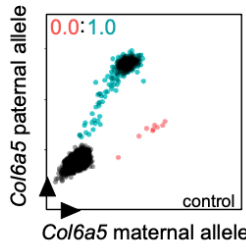

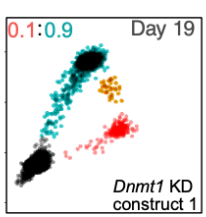

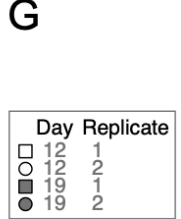

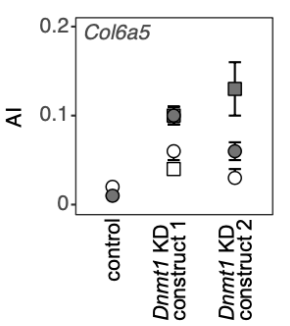

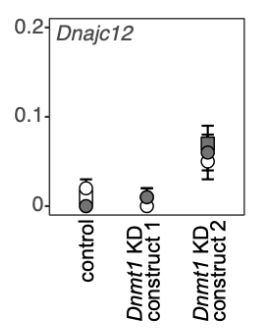

Figure 2. Hit validation for 5-aza-2'-deoxycytidine.

(A, B) Confirmation of Screen-seq results for 5-aza-2'-deoxycytidine (5-aza-dC) treated cells, using an orthogonal method to measure Al. cDNA samples from day 7 of screening were assessed using droplet digital PCR (ddPCR) with allele-specific fluorescent probes. (A) Scatterplots for 20,000 droplets targeting the readout gene, Col6a5. 5-aza-dC concentration is shown in the plots. Black: empty droplets; blue: droplets with the Cast paternal allele amplified (labeled by FAM fluorophore); red: droplets with the 129 maternal allele amplified (labeled by HEX fluorophore). Ratio of red:blue droplets shown. Red value is Al as used throughout the manuscript. (B) Left: Summary of Al measurements shown in (A) for Col6a5; right: summary of Al measurements for Dnajc12.

(C, D) Biological replicate of Abl.1 cells were treated with 5-aza-dC and Al was measured using ddPCR. (C) - Scatterplots as shown in (A) after 2 days of exposure. (D) - summary of Al measurement for Col6a5 (left) and Dnajc12 (right) after 2, 5, and 7 days of exposure. Grey vertical dashed lines for Col6a5 dose-response were used to determine "low", "medium" and "high" 5-aza-dC concentrations for the genome-wide experiments. Results for readout gene Adnp2 are in Suppl. Fig.S4. See also Suppl. Fig.S5-7. (E, F, G) Analysis of Dnmt1 knock-down (KD) in Abl.1 cells. (E) Real-time quantitative PCR (RT-qPCR) analysis of Dnmt1 relative expression (expression in the empty vector control, normalized to Nono, taken as 1.0). Abl.1 cells were transduced with an empty pIKO vector (control) or with 2 separate Dnmt1 shRNA knockdown constructs (Dnmt1 KD construct 1 or 2) and grown for 2 days. Transduced cells were then selected by growing in the presence of a selection antibiotic for an additional 17 days. RT-qPCR quantification was performed on cells collected 19 days after transduction. Mean and S.E.M for 3 technical replicates are shown. (F) Representative scatterplots show Al measurement for Col6a5 in the transduced Abl.1 cells. Al was measured using ddPCR. (G) Summary of the Al measurement for Col6a5 (left) and Dnajc12 (right) after Dnmt1 KD.

indicating that Al shifts are not a generalized feature of cells under stress.

To test if the effect of 5-aza-dC on allele-specific expression was specific to inhibition of methyltransferase activity, we assessed changes in $\mathrm{Al}$ in response to the knock-down of Dnmt1, the main maintenance methyltransferase in mammals (30). Abl.1 cells transduced with Dnmt1 shRNA constructs showed 2-fold and 4-fold decrease in Dnmt1 RNA abundance (Fig.2E), and the corresponding partial reactivation of silenced alleles of Col6a5 and Dnajc12 (Fig.2F,G).

Taken together, these observations indicate that Dnmt1-dependent DNA methylation is a molecular mechanism involved in Al maintenance for at least some MAE genes.

\section{Changes in allelic imbalance are long-term and rheostatic}

The shifts in Al we observe could be consistent with two different mechanisms. The shift could result from the long-term change in the mitotically stable state of allelespecific gene regulation. Alternatively, it could be due to short-term changes, e.g., because of stress caused by drug exposure. We thus asked if the changes in Al were mitotically stable and enabling long-term maintenance, the hallmark of autosomal MAE. 
To address this question, we performed a treatmentand-recovery experiment (Fig.3 and Suppl. Fig.S8A). First, Abl. 1 cells (with doubling time of $\sim 12$ hrs) were exposed to 5-aza-dC; after two days, cells were washed and incubated further in the regular growth medium. Fig.3A and 3B show the Al readout for Col6a5 gene (similar results were seen with Dnajc12 gene, Suppl. Fig.S8B). After two days of treatment and three days of recovery, Al reached levels that remained stable through days 9 and 12. This shows that Al shifts resulting from 5aza-dC treatment were maintained over multiple subsequent cell divisions. Such stability is consistent with DNA methylation as the molecular mechanism that maintains the long-term memory of Al state of MAE genes in clonal cells. A continuing Al shift over the first three days of recovery is consistent with the cell population right after treatment being heterogeneous and containing some remaining fraction of cells with the readout gene in the initial state of $A l \approx 0$. By day 5 , that fraction would be replaced by cells in the new stable state of DNA methylation, and the new state would then be maintained through days 9 and 12 .

We observed in other experiments (see Fig.2) that the extent of allelic shift was dose dependent. Notably, after recovery, the eventual stable Al states were also dependent on the 5-aza-dC concentration during cell exposure (Fig.3). This shows that 5-aza-dC-dependent allele-specific regulation acts not as an on-off switch, but rather as a rheostat, with multiple stable intermediate states.

Similarly, the extent of the Al shift correlated with the length of drug exposure. After two days of exposure, half the cells were moved into the regular growth medium, while the other half were exposed to the same drug concentration for an additional 3 days. Additional exposure led to further Al shifts (Suppl. Fig.S8A). Note that longer exposure to 5 -aza-dC decreased cell viability to the point where insufficient number of viable cells were present after day 5 for reliable analysis (Suppl. Fig.S8C). The same eventual Al shifts were reached by cells after 5 days of treatment as after treatment and recovery: $\mathrm{Al} \approx 0.9$ was reached after (i) two days of treatment with $1 \mu \mathrm{M} 5$ aza-dC with three days recovery; (ii) five days treatment with $0.5 \mu \mathrm{M}$; and (iii) five days treatment with $1 \mu \mathrm{M}$ (Suppl.

Fig.S8B). This is consistent with a regulatory locus reaching complete demethylation in all conditions.

\section{Genome-wide allele-specific impact of DNA demethylation}

We assessed the global impact of 5-aza-dC on allelespecific DNA methylome and transcription by exposing Abl. 1 cells for 2 days to low $(0.2 \mu \mathrm{M})$, medium $(2 \mu \mathrm{M})$ or high $(10 \mu \mathrm{M})$ concentrations of 5-aza-dC, compared with solvent only (1\% DMSO) as the control, and performing RNA and reduced-representation bisulfite (RRBS (31)) sequencing. When not resolving allele-specific signal,
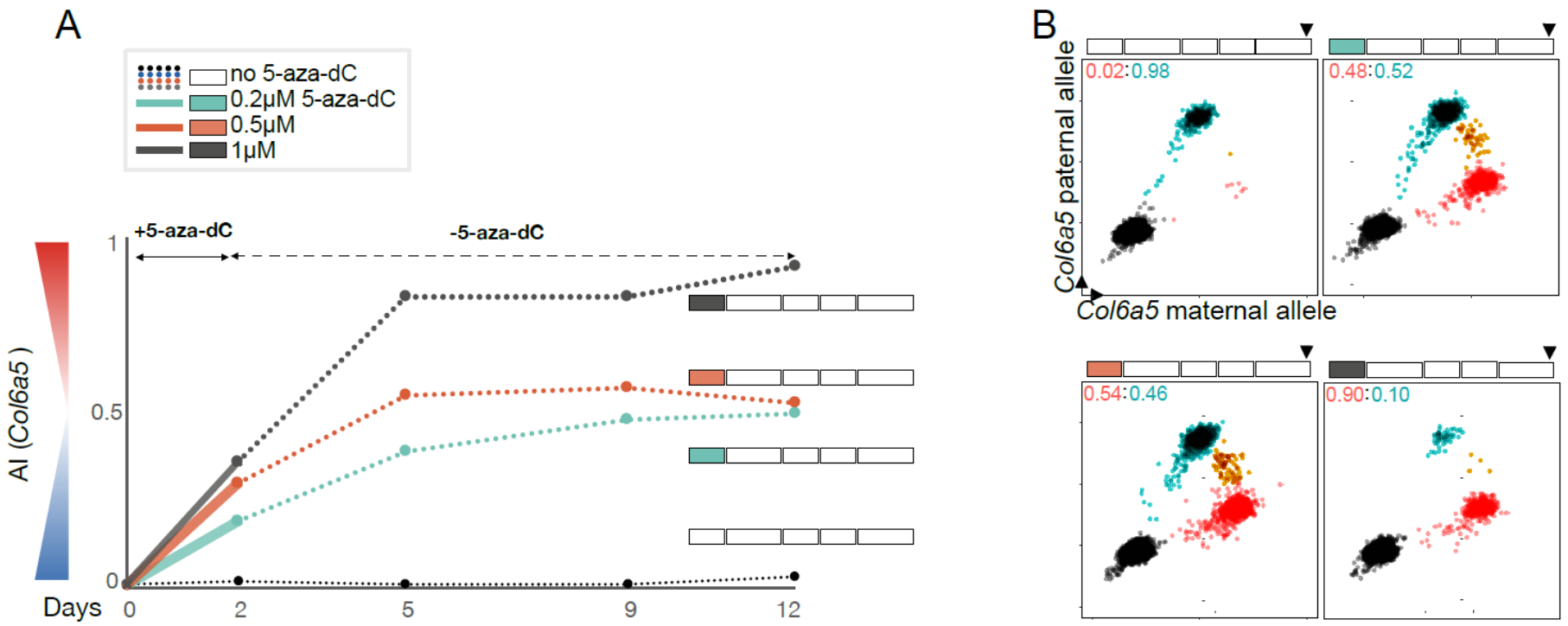

Figure 3. Long-term changes in the mitotic memory of allelic imbalance after exposure to 5-aza-dC and recovery.

(A) 5-aza-dC exposure/recovery experiment in Abl.1 cells. Drug treatment setup is shown next to the line-plots (small box- 2 days, long box - 3days). Media changes are shown as breaks in the boxes in the drug treatment setup. Cells were exposed to 0.2 , 0.5 or $1 \mu \mathrm{M} 5$-aza-dC (denoted by colors) in growth medium. Cells were moved to the medium without any drug after 2 or 5 days, and collected for analysis at days 2, 5, 9, and 12. Al measurements for Col6a5 across time points using ddPCR are summarized in the line-plot. Results shows here are for cells that were exposed to 5-aza-dC for 2 days. See Suppl. Fig. S8 for the complete results.

(B) ddPCR scatterplots for Col6a5 on day 12 (shown by arrows on the drug treatment setup) after recovery [as summarized in (A)]. 
both RNA abundance and the extent of DNA methylation behaved as expected. DNA methylation levels substantially decreased in the presence of 5-aza-dC (Suppl. Fig.S9). Consistent with the overall transcriptional derepression due to DNA demethylation, RNA abundance generally increased (Suppl. Fig.S10).

To resolve allele-specific signal in transcription, we applied Qllelic, a novel, highly stringent approach to the analysis of allele-specific expression, which uses replicate RNA-seq libraries to account for technical Al overdispersion and to minimize false positives for differential Al (22). Note that accounting for the wholetranscriptome scale of RNA-seq necessarily made some Al changes, readily detectable in targeted assays, fall short of statistical significance in RNA-seq. For example, of the three genes with shifts in 5-aza-dC detected with
A

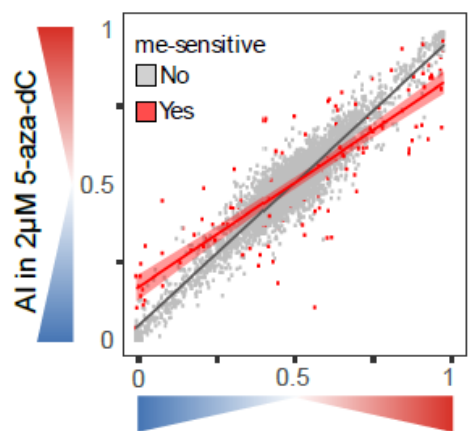

$\mathrm{Al}$ in DMSO

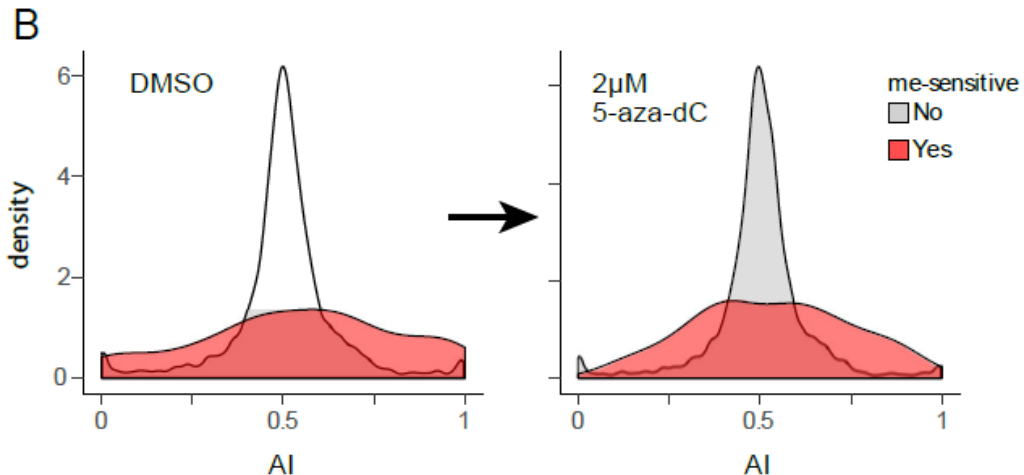

$\mathrm{Al}$
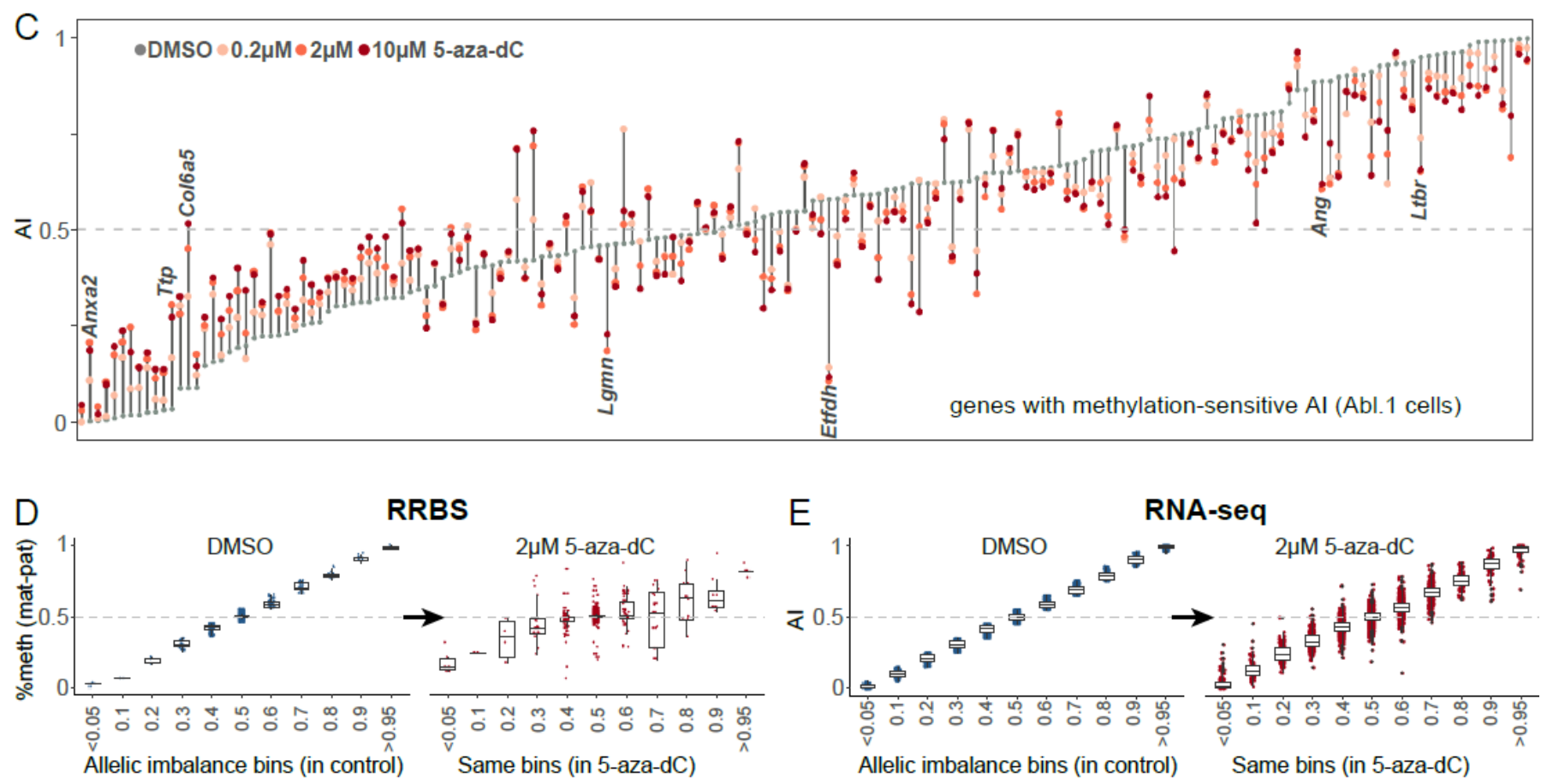

E

RNA-seq

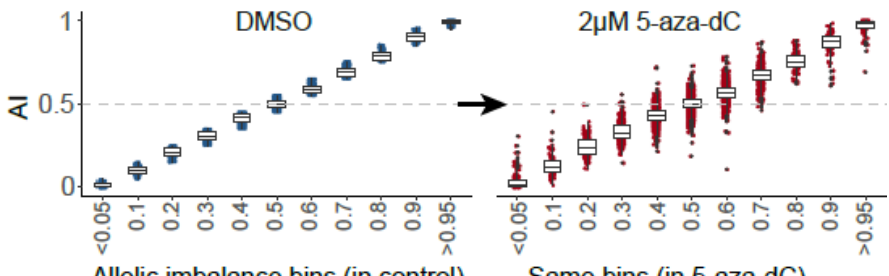

Figure 4. Genome-wide allele-specific effects of cell exposure to 5-aza-dC.

(A) Comparison of allele-specific expression in Abl.1 cells in control cells and $2 \mu \mathrm{M} 5$-aza-dC. Genes with significant shift in Al [methylation-sensitive genes] are shown in red. For other 5-aza-dC concentrations, see Suppl. Fig.S11.

(B) Density plots for distribution of Al values of genes with no significant changes in Al (grey) and with changes (red) [same experiment as in (A)]. Note that grey and red areas are plotted to be equal. Left: control; right: $2 \mu \mathrm{M} 5$-aza-dC. For other concentrations, see Suppl. Fig.S19.

(C) Shifts of Al in expression for methylation-sensitive genes [same experiment as in (A)]. Genes shown in the order of their nominal $\mathrm{Al}$ in control (grey circles). Al after treatment is shown (color denotes 5-aza-dC concentration). For other clonal lines, see Suppl. Fig.S15. For full list of methylation-sensitive genes, see Suppl. Table S7 and Suppl. Fig.S12.

(D-E) Allele-specific changes in DNA methylome and transcriptome in Abl.1 cells. (D) Allele-specific DNA methylation analysis of RRBS (reduced representation bisulfite sequencing) data. Left: Sites were binned by Al, defined as difference between methylated fraction of CpGs on maternal and paternal alleles in control conditions (1\% DMSO). Right: Al in the same bins after treatment. See also Suppl. Fig.S9. (E) Genes were binned by Al values, in control (left) and after treatment (right). Note: in (D,E), Al bins are labeled with central value $X$, with the bin covering the range of $(X-0.05 ; X+0.05]$. See also Suppl. Fig.S10 and $\mathbf{S 1 3}$ for bins showing significant shifts between control and treatment. 

available under aCC-BY-NC-ND 4.0 International license.

Screen-seq (Fig.1) and confirmed using ddPCR (Fig.2), only the shift in Col6a5 reached statistical significance in the RNA-seq analysis (Suppl. Table S6). This underscores the utility of targeted assays for screening purposes.

Applying this stringent analysis to the Abl.1 RNA-seq data, we saw 51 genes with a significant Al shift after exposure to the low 5-aza-dC concentration, 145 genes in medium, and 140 genes in high 5-aza-dC (Fig.4A, Suppl. Fig.11 and Suppl. Table S7). The direction of Al shifts at different concentrations of 5-aza-dC all agreed, with higher concentration generally corresponding to larger shift (Fig.4C). No known imprinted genes showed Al changes under these conditions, and for the $3 \mathrm{X}$ linked genes with statistically significant changes, the absolute shift was very small (e.g., shift in Hccs from $\mathrm{Al}=0.0$ to 0.05 , Suppl. Table S7), suggesting more robust mitotic maintenance of imprinting and Xinactivation than MAE.

Known examples of changes in transcription $\mathrm{Al}$, such as loss of imprinting and loss of $\mathrm{X}$-inactivation in cancer $(32,33)$ involve relaxation of very strong allelic biases towards $\mathrm{Al}=0.5$. This aligns with the notion of genetic variation by itself making a small contribution to allelic bias, while epigenetic mechanisms can impose dramatic allelic imbalance. In Abl.1 cells, observed initial Al values and shifts were following this pattern in some genes (e.g., Anxa1, Ttp, Ang, and Ltbr; Fig.4C)).

Use of replicate RNA-seq libraries and Qllelic enabled highly confident estimation of $\mathrm{Al}$, revealing that most of the genes with Al shifts had initial (before treatment) Al values between extreme bias and $A l=0.5$, with these intermediate values much more common among these genes than in the transcriptome as a whole (Fig.4B). In addition, the direction of the Al shifts was also often unexpected. Out of 145 genes with significant shifts in Al, 44 genes (30\%), including Lgmn and Etfdh, showed greater allelic bias (further away from $\mathrm{Al}=0.5$ ) after 5 -aza$\mathrm{dC}$ exposure than in their initial state (at $2 \mu \mathrm{M} ; 13 / 51$ genes $(26 \%)$ at $0.2 \mu \mathrm{M}$, and $37 / 140(26 \%)$ at $10 \mu \mathrm{M}$; Fig.4C).

To analyze changes in allele-specific signal in the DNA methylome from RRBS data, we first grouped sites by Al bins, since very few individual sites had sufficient coverage for statistical significance in a genome-wide analysis (Suppl. Table S9). In this analysis (Fig.4D), every bin with significant Al changes showed a shift towards $\mathrm{Al}=0.5$ (Suppl. Fig.S13A). This appears inconsistent with $\sim 30 \%$ of the genes showing increased $\mathrm{Al}$ in expression after exposure to 5-aza-dC (Suppl.

Fig.S13B and Fig.4C). Furthermore, using similar binning of genes by $\mathrm{Al}$, although the overall relaxation towards $\mathrm{Al}=0.5$ was statistically detectable, the extent of this Al shift was much less pronounced than for RRBS (Fig.4E).

Taken together, these observations show that $\mathrm{Al}$ in transcription is not necessarily a simple predictor of $\mathrm{Al}$ in DNA methylation. Instead, allele-specific gene expression is often determined by an interplay of genetic and epigenetic regulation.

\section{DNA demethylation leads to increased similarity between clones}

An MAE gene can show extreme allelic bias in one clone and biallelic expression in another; this is a distinct feature of MAE genes, in contrast to $\mathrm{XCl}$ and imprinting $(5,34)$, and it directly contributes to clonal heterogeneity. In previous RNA-seq studies of MAE, assignments of allelic states were categorical and based on arbitrary thresholds. Gene expression would be classified as "monoallelic" if the major allele constitutes over $85 \%$ (9), or $66 \%(12)$, or $98 \%(7)$.

We took advantage of the precision of Al estimates from Qllelic to perform differential analysis of allelespecific expression across several clones and its changes after DNA demethylation. In addition to Abl.1 clone, we assessed allele-specific expression in clones Abl.2, Abl.3, and Abl.4, all derived from 129xCastF1 mice (13) and thus genetically nearly identical. To control for possible loss of heterozygosity events, we performed exome sequencing and removed from comparisons any locus with pronounced allelic bias in genomic DNA ( $\mathrm{Al}<0.3$ or $\mathrm{Al}>0.7$; see Methods).

To compare shifts in Al after DNA demethylation, we first assessed the toxicity of 5-aza-dC across clones. The viability of Abl.2, Abl.3, and Abl.4 clones was affected at $0.2 \mu \mathrm{M}$ similarly to the Abl. 1 clone at $2 \mu \mathrm{M}$ (Suppl. Fig.S14). After two days' exposure to these equitoxic concentrations, 677 genes between the four assessed clones showed Al shifts: 252 genes in Abl.2, 282 in Abl.3, and 172 in Abl.4 cells (Suppl. Table S7). Al shifts had similar features in all clones. Initial and final Al states of the affected genes were distributed similarly to Abl.1 clone, and the direction of shifts was also similar (Suppl. Fig.S15). 
There were 1,767 genes with significant differential Al between clones (Suppl. Table S11), and $346(20 \%)$ of these showed significant changes of Al after DNA demethylation (Suppl. Table S12). Al in the other genes with differential Al did not change after exposure to 5aza-dC, suggesting that the mitotic maintenance of differences in Al between clones in such loci was due to some mechanism other than DNA methylation.

Comparison of $\mathrm{Al}$ changes of the same genes across clones revealed a striking property. Depending on the initial Al, allelic bias in a given clone could shift towards balanced expression $(\mathrm{Al}=0.5)$ or towards stronger bias. However, when considered together, Al values converged across clones after exposure to 5-aza-dC (Fig.4F). Principal component analysis for all genes with Al shifts showed that transcriptome-wide Al states of clones became closer to each other after demethylation (Suppl. Fig.S16). The target Al to which clones converged varied between genes and encompassed the whole range of $\mathrm{Al}$ values, including extreme allelic bias for some genes (inferred target values shown as green boxes in Fig.5 and Suppl. Fig.S17). In clones where an MAE gene was already in the target Al state (e.g., Col6a5 in Abl.3 and Abl.4, or Casp6 in Abl.1 and Abl.4; Fig.5), there were no further Al shifts after DNA demethylation.
These observations are consistent with a simple model in which the Al for many MAE genes are set at different values across clones and are maintained via DNA methylation of regulatory sequences in cis to the affected genes. Demethylation causes these Al values to converge to a genetically determined "target" state, with partial demethylation resulting in an Al value between the start and the target states (Fig.5).

\section{DISCUSSION}

Using a screening-by-sequencing approach, we identified DNA methylation as a key mechanism involved in the mitotic maintenance of monoallelic expression in clonal cell lineages of mammalian cells. Dnmt1dependent maintenance DNA methylation offers a straightforward explanation for MAE stability, since it is a very stable form of molecular memory: cytosine methylation in the Cryptococcus genome has apparently been maintained for millions of years in the absence of de novo methylation (35). We propose a simple model (Fig.5), with the allele-specific regulatory landscape defined by genetic variation (possibly in interaction with epigenetic mechanisms), while a specific state of a clonal cell population depends on DNA methylation. Note that this model predicts specific regulatory elements located in cis to the affected genes. Such genomic elements would offer a simple explanation to
A

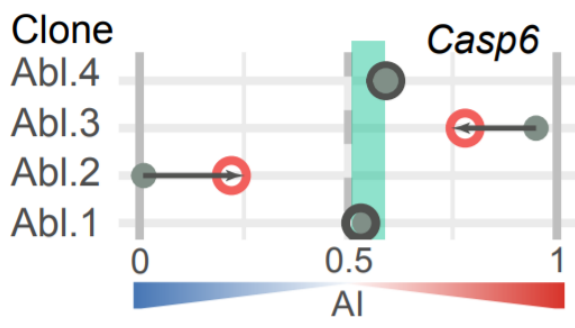

D high
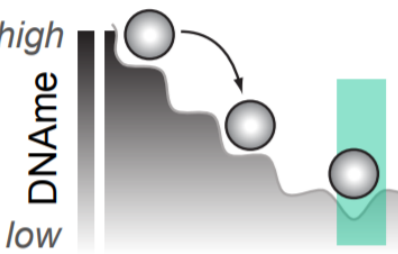

- Al in control
B

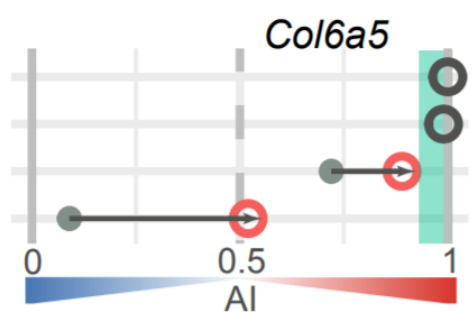

E

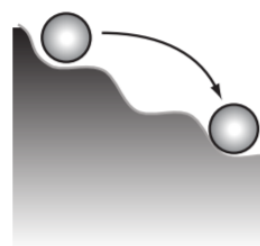

C

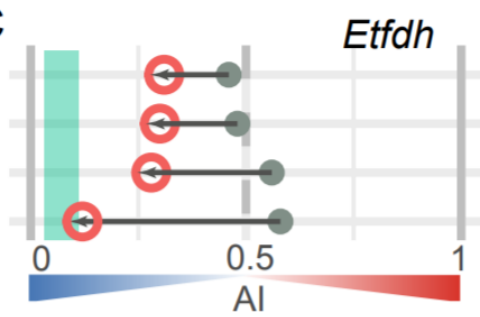

F

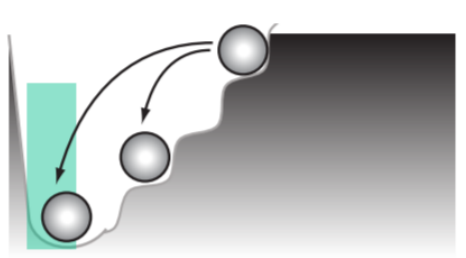

Figure 5. DNA methylation as a rheostatic mechanism of the mitotic maintenance of allele-specific expression.

(A-C) Examples of genes with significant changes in Al across four lymphoid clonal lines. All genes with statistically significant shifts (after correction for Al overdispersion) are shown in Suppl. Fig.S17. Al values in control (filled grey circles) and after exposure (open circles) are shown (no shifts are in grey). Same Abl.1 data as in Fig.4. Clones Abl.2, Abl.3 and Abl.4 were exposed to 0.2 $\mu$ M 5-azadC. Green box denotes imputed common end states.

(D-F) Diagram of interaction between cis-regulatory landscape and DNA methylation (DNAme) which determines the rheostatic control of allele-specific expression. There are multiple stable DNAme states, and corresponding Al in expression. Decreases in DNAme lead (arrows) to convergence of states between clones, with complete loss of DNAme leading to common Al state (green box). 
bioRxiv preprint doi: https://doi.org/10.1101/2020.02.20.954834; this version posted July 23, 2020. The copyright holder for this preprint (which was not certified by peer review) is the author/funder, who has granted bioRxiv a license to display the preprint in perpetuity. It is made available under aCC-BY-NC-ND 4.0 International license.

the evolutionary conservation of MAE status of genes across human populations (18) and between human and mouse $(12,13,15)$. When and how DNA methylation is established in these regulatory regions remain to be uncovered.

Not all assessed MAE genes were affected by DNA demethylation, suggesting that MAE maintenance for some loci involves other mechanisms instead of (or in addition to) DNA methylation. This offers one likely explanation of the previous observations that DNA demethylating agents did not affect allelic imbalance in any of the several assessed MAE genes $(8,9)$.

Consistent with the idea of additional mechanisms of MAE maintenance, a SIRT1 activator BML-278 and a sirtuin inhibitor, salermide, appeared as primary hits in our screen (see Suppl. Fig.S3), suggesting that expanded application of the Screen-seq strategy can uncover such additional mechanisms.

We assessed the genome-wide impact of DNA demethylation on the allelic imbalance in the transcriptome of clonal lymphoid cells. Using a new, highly stringent approach to allele-specific RNA-seq analysis (22), we found Al shifts in over 600 autosomal genes between four analyzed clones. Interestingly, only six X-linked genes showed small but statistically significant changes (Suppl. Fig.S18), suggesting that DNA methylation-dependent mitotic maintenance of $\mathrm{Al}$ is easier affected in autosomal genes than X-chromosome inactivation.
Significant impact of DNA demethylation drugs on allele-specific expression in lymphocytes is of particular importance since both 5-aza-2'-dC and 5-azaC are used in the clinic to treat acute leukemia and other malignancies (28). Notably, concentrations of these compounds in our experiments $(0.2-1.0 \mu \mathrm{M}$; Fig.3) are similar to that measured in the patients' plasma [5-aza$\mathrm{dC}$ at $\sim 60 \mathrm{ng} / \mathrm{ml}$, about $0.25 \mu \mathrm{M}(36)]$. Our findings thus imply that DNMT inhibitors likely affect gene regulation in patients in ways that would be undetectable without allele-specific analysis. Similarly, we note that large shifts in Al were often independent of changes in overall RNA abundance (see Suppl. Fig.S10, Suppl. Fig.S12 and Suppl. Table S8). This suggests that analyses of allele-specific gene regulation in polyclonal and monoclonal cell populations should lead to new translational insights.

Stringent quantitative analysis of RNA-seq data reveals a more complex landscape of mitotically stable clonal diversity in allele-specific gene regulation than is implied by monoallelic/biallelic dichotomy. This complexity suggests that the biologically relevant questions concern molecular mechanisms and functional impact on cellular function, rather than arbitrary thresholds. In different clones, Al of a gene can span a range of values, with epigenetic regulation acting as a rheostat rather than an on/off switch. This extends the idea of the rheostatic role of DNA methylation, proposed for the regulation of the overall abundance of transcripts $(37,38)$. 


\section{METHODS}

\section{Cell culture}

v-Abl pro-B clonal cell lines Abl.1, Abl.2, Abl.3 and Abl.4 (13) were cultured in Roswell Park Memorial Institute medium (Gibco), containing 15\% FBS (Sigma), 1X L-Glutamine (Gibco), 1X Penicillin/Streptomycin (Gibco) and $0.1 \% \beta-$ mercaptoethanol (Sigma).

\section{Drug treatment}

The SCREEN-WELL® Epigenetics arrayed drug library was purchased from Enzo Life sciences (BML-2836). The Abelson clone Abl.1 was treated with the entire drug library (Suppl. Table S3) at concentrations of $1 \mu \mathrm{M}, 10 \mu \mathrm{M}$ and $20 \mu \mathrm{M}$ in order to encompass a wide enough range of concentrations where the drugs are potentially pharmacologically active. Cultures were treated for 21 days where media was changed every second day. After following up hits from the initial drug screen, 5-aza-2'-deoxycytidine (5-aza-dC, Sigma, A3656) was diluted in DMSO at a concentration of 10mM and Abl.1 cells were treated using a concentration range of $10 \mathrm{nM}$ to $20 \mu \mathrm{M}$ 5-aza-2'-deoxycytidine. Cells were treated for a total of 21 days where media was changed every 2 days and samples of $\sim 1 \times 10^{5}$ cells were harvested for RNA extractions on days $2,5,7$, 9, 12 and 14. Viable cells were counted using trypan blue solution (Gibco ${ }^{\mathrm{TM}}$ ) on Countess ${ }^{\mathrm{TM}}$ II FL Automated Cell Counter machine (Life Technologies). For all treatments, drugs were solubilized in DMSO and dilutions were made to ensure the final DMSO added to cultures was 1\% (v/v). For exposure/recovery experiment (Fig.3), 5-aza-dC was dissolved in water.

\section{RNA and DNA preparation}

For all Abelson monoclonal cultures, RNA was extracted from cells using a magnetic bead-based protocol using Sera-Mag SpeedBeads $^{T M}$ (GE Healthcare). Isolated RNA was DNase-treated with RQ1 DNase (Promega). First strand cDNA synthesis was done using Episcript ${ }^{\mathrm{TM}} \mathrm{RNase} \mathrm{H}$-reverse transcriptase (Epicentre) where RNA samples were primed with random hexamers (NEB). Both DNase treatment and cDNA synthesis were performed using manufacturer specifications with minimal modifications. For RNA preparation from mouse spleen, cells were extracted by crushing the whole spleen using the back of $1 \mathrm{ml}$ syringe plunger in $40 \mu \mathrm{M}$ nylon filter and washing the strainer with 1X PBS (Phosphate-buffered saline, Sigma) to collect cells. Cells from spleen were spun down and RNA was extracted using Trizol reagent (Invitrogen). Genomic DNA extractions for testing the sensitivity of Screen-seq were performed using the salting out method (39) and for reduced representation bisulfite sequencing (RRBS) were performed using Sigma GenElute kit (G1N10-1KT). RT-qPCRs were performed using iTaq ${ }^{\text {TM }}$ Universal SYBR® Green Supermix (BioRad) using manufacturer's protocol on a 7900HT Fast Real-Time PCR system (Applied Biosystems Inc.). All primers used in this study were ordered from Integrated DNA Technologies and their sequences are listed in Suppl. Table S2.

\section{Screen-seq methodology}

A targeted sequencing method similar to that described in Nag et al (2013), was used to assay multiple genes simultaneously for assessing allele-specific expression. Here, we assayed 23 genes. The assay involved RNA extraction, cDNA synthesis (Fig. 1B), two rounds of PCR amplification and Illumina sequencing. After magnetic bead-based RNA purification, cDNA synthesis was performed within each well of a 96-well plate, separately using EpiScript ${ }^{\mathrm{TM}}$ Reverse Transcriptase (EpiCentre Biotechnologies) using both random hexamers (NEB) and UMI-tagged oligo-dT primer with universal tail (Suppl. Table S2) using manufacturer's instructions. Half the portion cDNA products were transferred to a separate 96-well plate. Gene-specific multiplex PCR are performed in both the plates using Phusion U multiplex Master Mix (ThermoFisher, F562L, Waltham, MA). Two types of multiplexed readouts were generated within each plate: readouts without UMI and readouts with 3'-UMI. For the multiplex readouts without UMI, target genes that contain the SNP(s) differentiating the maternal and paternal allele, were amplified using gene-specific primer pairs containing one of two universal tails (UT1 or UT2, Suppl. Table S2). For the multiplex readouts with 3'-UMI, the forward primers were genespecific and contained universal tail UT2 (Suppl. Table S2). They were always positioned near the SNP of interest. Reverse primer for these genes were complimentary to the universal tail UT1. These readouts were always constrained to the 3' end of the transcript. These two types of multiplex readouts were not generated for all readout genes. A list the readout genes for which the multiplex assay was used is given in Supp. Table S1. MPprimer primer design program (40) was used to design the non-UMI multiplex PCR assay. We computationally generated an input form that would 1) constrain our SNP(s) of interest within 135 base pairs from one end of the amplicon, 2) mask repetitive regions, 3) prevent the design of primers 
pairs that exist within more than one exon and 4) ensure that the total fragment size for each readout falls within 250-500 base pairs. Once the gene-specific primer sequences were designed, the universal tails were added. Primers generated were tested for specificity and primer dimerization using MFEprimer (41) and also experimentally validated. The two groups of multiplex products from the gene-specific PCR were combined and carried over as templates to the second PCR which is performed using Phusion ${ }^{\circledR}$ High-Fidelity DNA Polymerase (New England Biolabs Inc., M0530L, Ipswich, MA) that barcodes each well/perturbation separately. These reactions use primers that target the universal tails (UT1 and UT2) of the readouts amplified in the first multiplex PCR and add a six-nucleotide barcode, a seven-nucleotide spacer and an Illumina primer dock (Suppl. Table S2). Combinatorial barcoding was achieved by using a pair of unique forward and reverse primers, which tag each sample with a unique barcode combination. These barcode combinations allowed pooling of samples in the subsequent steps of the assay. Once pooled, the readout library was cleaned up using magnetic beads at a bead to sample ratio of 1.2 to get rid of primer dimer bands $<150 \mathrm{bp}$ in size. The sample was then carried over as a template into a third PCR reaction which adds Illumina adapters.

We observed high accuracy of multiplexing and barcoding steps of Screen-seq by comparing the Al calculated from Screenseq and expected Al for a range of pure 129 and Cast parental genomic DNA mixes for all genes (Suppl. Fig.S1). A good correlation was observed between the reads with UMI and without UMI for readout genes tested using both methods. For this, Screen-seq was performed for a range of RNA mixes from pure 129 and Cast mice spleen. Allelic imbalance (AI) calculated from Screen-seq for Adamts/4 and Adnp2 showed good correlation with the expected AI, and also between UMI and non-UMI assays (Suppl. Fig.S2). Smtn/2 and Dnajc12 showed low expression in mice spleen tissue and hence comparison could not be made. Finally, the assays for genes we selected had to combine successfully in multiplexed PCR.

\section{Screen-seq data analysis}

After Screen-seq libraries were prepared as described above, they were sequenced at the UMass Boston and Center for Cancer Systems Biology (CCSB) sequencing core on Illumina HiSeq 2500 and MiSeq, respectively using four-color reagent kits. From the P7 adapter end, 65nt were sequenced (Read 1), including one of the two barcodes for encoding plate wells (and the UMI, where appropriate). From the P5 adapter the remaining 135nt were sequenced (Read 2), covering the second well-encoding barcode and the cDNA amplicon containing the interrogated SNP. In addition, standard Illumina barcodes were used to distinguish individual plates within the overall pooled library, with demultiplexing before further processing. Reads were aligned using bowtie2 (42) against mm10 mouse genome assembly. The resulting BAM files were processed using custom Perl scripts to extract allele-specific, UMI-corrected counts for each gene and each well.

To identify primary hits (outliers in Figs.1E and 1F), the allele-specific counts were analyzed using custom R scripts. Briefly, for each gene, point Al estimates for all drug conditions were considered together to determine median $\mathrm{Al}$ and the interquartile range (IQR = Q3 - Q1, with Q1 and Q3 the 25th and 75th percentiles). Observations with counts under 50 were filtered out (an observation consists of allelic counts for one gene in one well). A common practice for identification of outliers is to use values below Q1-1.5×IQR or above Q3+1.5×IQR (44). We used a more stringent threshold of $3 \times 1 \mathrm{QR}$, to reduce the likelihood of false positive hits. Complete results can be found in Suppl. Table S5.

\section{Droplet Digital PCR}

Droplet digital PCRs (ddPCRs) were performed on QX200 ddPCR system (BioRad) for absolute quantification of 129 and Cast alleles using manufacturer-recommended settings. C1000 Touch ${ }^{\mathrm{TM}}$ thermal cycler was used to perform amplification within droplets. SNP-specific TaqMan assays (IDT; sequences in Suppl. Table S2) were designed manually. We first validated all TaqMan assays experimentally using homozygous Cast and $129 \mathrm{cDNA}$ and optimized reaction conditions for each assay using Abl.1 clonal cell line cDNA, including Tm of each primer-probe mix by performing thermal gradient PCR. Finally, we tested the specificity of this method by using known quantities of left kidney cDNA from homozygous 129 and Cast mice parents and comparing it with the estimated allelic imbalance from ddPCR. To determine the false-positives, we made 2-fold dilutions of these samples starting from $1 \mathrm{ng}$ cDNA till its $1 / 16^{\text {th }}$ dilution. Results demonstrated our ability to precisely measure allelic imbalance in samples with 30 copies/ $\mu$ using ddPCR. CDNA was prepared from around 100,000 cells and $8 \mu \mathrm{l}$ template cDNA ( $1 / 4^{\text {th }}$ of eluted sample) was used per reaction. Gating for clusters with maternal and paternal alleles was decided by comparing the fluorescence intensity individually for the maternal and paternal probes in homozygous 129 and Cast tissue samples. Data was processed using QuantaSoft v.1.6 (Bio-Rad). Inverse fractional 
abundance given displayed by the Quantasoft software was divided by 100 and noted as Al measurement [mat/(mat+pat)] from ddPCR.

\section{ShRNA infection}

Two shRNA vectors targeting Dnmt1 (SHR000038801.1_TRC001.1 and SHR000373188.1_TRC005.1) and a control empty vector (NUL003.3_TRC021.1) packaged in lentiviral vectors obtained from the Genetic Perturbation Platform at the Broad Institute were tested. The optimal multiplicity of infection (MOI) was determined by infecting Abl.1 cells with pLKO_TRC060 lentiviral vector expressing eGFP. Abl.1 cells were infected with 3 shRNA vectors (2 targeting Dnmt 1 and 1 control) individually on day 1 at the optimal $\mathrm{MOI}$ under normal growth conditions in the presence of $8 \mu \mathrm{g} / \mathrm{ml}$ polybrene and spun at $800 \times \mathrm{g}$ for 90 minutes at $37^{\circ} \mathrm{C}$. The next day the media was changed and media containing $2 \mu \mathrm{g} / \mathrm{ml}$ of puromycin was added on day 2. Selection was maintained continuously afterwards, and media changes were done every 2-3 days. Cells were harvested on day 12 and 19 after infection, and RNA was extracted.

\section{Estimation of allelic imbalance in RNA-seq, RRBS and exome sequencing.}

$5 \times 10^{6}$ cells treated with concentrations of $0.2 \mu \mathrm{M}, 2 \mu \mathrm{M}$ and $10 \mu \mathrm{M} 5$-aza-2'-deoxycytidine were harvested on days 1,2 and 5 . Live cells were separated from debris by sucrose gradient centrifugation (Histopaque $®-1077$, Sigma). RNA was extracted from $2 \times 10^{5}$ live cells, and the remaining live cells were washed with 1 X PBS and flash frozen on dry ice for genomic DNA extractions.

Libraries for RNA-seq were prepared for cells collected on day 2, using at least two technical replicates from the same RNA (5 replicates for Abl. 1 cells treated with $2 \mu \mathrm{M}$ 5-aza-dC), using SMARTseqv4 kit (Clonetech), starting with 10 ng input RNA for each library according to manufacturer's instructions. Library preparation, QC and sequencing were performed at the Molecular Biology Core Facilities at Dana-Farber Cancer Institute. Single-end 75bp reads were generated using a Nextseq 500 instrument (Illumina).

Allele-specific gene expression analysis was performed using ASEReadCounter* and Qllelic version v0.3.1 pipeline described in (22). Briefly, RNA-seq reads were aligned with STAR aligner v.2.5.4a using imputed parental genomes as reference, with default quality filtering. Only uniquely aligned reads were used. Allele-specific coverage over SNPs was counted using samtools mpileup and further processed using ASEReadCounter* tool based on the GATK pipeline. All exons belonging to the same gene were merged into a single gene model based on RefSeq GTF files (GRCm38.68 and GRCh37.63); overlapping regions that belong to multiple genes were excluded. Al point estimate per gene obtained as a proportion of maternal gene counts to total allelic gene counts. Differences in Al were accepted as significant after accounting for experiment-specific overdispersion, estimated using Qllelic v0.3.1 analysis of replicate libraries. Complete results can be found in Suppl. Table S9.

To control for the possible loss of heterozygosity, exome sequencing was performed on genomic DNA for all clones. Library preparation, QC and sequencing (50x) were performed at LC Sciences (TX, USA). Exome capture was performed using SureSelect (Agilent Technologies) following the vendor's recommended protocol. Paired-end 150bp reads were generated using a Hiseq X Ten sequencing instrument (Illumina). Genes with total allelic counts of $<10$ and those with nominal $\mathrm{Al} \geq 0.7$ or $\leq 0.3$ were excluded from respective clones before comparing RNA-seq data between clones.

For Reduced Representation Bisulfite-seq (RRBS), libraries were generated from 50 ng input genomic DNA using a scaleddown (half reactions) of the NuGEN Ovation RRBS Methyl-Seq System (Tecan) following the manufacturer's recommendation. Libraries were PCR amplified for 11 cycles. Paired-end 100bp reads were generated using HiSeq 2500 instrument (Illumina). Reads were aligned to the mouse mm10 genome using BSmap3 with flags -v 0.05 -s 16 -w 100 -S 1 -p 8 -u. Custom scripts written in Perl were used to calculate the methylation percentage for CpGs covered by 4 or more reads (43) at locations of known SNPs. Briefly, VCF files containing SNPs between 129 and Cast strains were filtered to exclude calls that did not pass minimum requirements as well as $C \rightarrow T$ or $G \rightarrow A$ calls. For each SNP, RRBS reads that overlapped that SNP were extracted, and the methylation status of genomic cytosines was calculated by dividing the number of unconverted (methylated) cytosines $(C)$ by the total number of unconverted $(C)$ or converted $(T)$ cytosines. The methylation status of all cytosines on reads overlapping a SNP were aggregated by SNP status to create a methylation average for the reference and alternate genotype. Complete results can be found in Suppl. Table S10. 
bioRxiv preprint doi: https://doi.org/10.1101/2020.02.20.954834; this version posted July 23, 2020. The copyright holder for this preprint (which was not certified by peer review) is the author/funder, who has granted bioRxiv a license to display the preprint in perpetuity. It is made available under aCC-BY-NC-ND 4.0 International license.

\section{Data availability}

RNAseq and RRBS data is deposited at NCBI Gene Expression Omnibus (GEO) repository under accession number GSE144007 (subseries: GSE144005 for RNAseq and GSE144006 for RRBS).

\section{ACKNOWLEDGMENTS}

We thank Alex Bortvin, Arun Chavan, Mary Gehring, David Hill, Mitzi Kuroda and members of the Gimelbrant lab for valuable comments, Matt Warman for generously sharing a ddPCR system, and Kim Blanchard and Arman Mohammed for technical help.

This work has been supported by $\mathrm{NIH}$ grants R21HD081675 and R01GM114864 to AAG and Marie Curie fellowship (EU project 752806 ) to CFAP. 


\section{REFERENCES}

1. Mohammadi $P$, Castel SE, Brown AA, Lappalainen T (2017) Quantifying the regulatory effect size of cis-acting genetic variation using allelic fold change. Genome Research 27(11):1872-1884.

2. Tucci V, Isles AR, Kelsey G, Ferguson-Smith AC, Erice Imprinting Group (2019) Genomic Imprinting and Physiological Processes in Mammals. Cell 176(5):952965.

3. Galupa R, Heard E (2018) X-Chromosome Inactivation: A Crossroads Between Chromosome Architecture and Gene Regulation. Annu Rev Genet 52(1):535-566.

4. Chess A, Simon I, Cedar H, Axel R (1994) Allelic inactivation regulates olfactory receptor gene expression. Cell 78(5):823-834.

5. Gimelbrant A, Hutchinson JN, Thompson BR, Chess A (2007) Widespread monoallelic expression on human autosomes. Science 318(5853):1136-1140.

6. Chess A (2016) Monoallelic Gene Expression in Mammals. Annu Rev Genet 50(1):317-327.

7. Deng Q, Ramsköld D, Reinius B, Sandberg R (2014)

Single-cell RNA-seq reveals dynamic, random monoallelic gene expression in mammalian cells. Science 343(6167):193-196.

8. Eckersley-Maslin MA, et al. (2014) Random Monoallelic Gene Expression Increases upon Embryonic Stem Cell Differentiation. Developmental Cell 28(4):351365.

9. Gendrel A-V, et al. (2014) Developmental dynamics and disease potential of random monoallelic gene expression. Developmental Cell 28(4):366-380.

10. Jeffries AR, et al. (2012) Stochastic choice of allelic expression in human neural stem cells. Stem Cells 30(9):1938-1947.

11. Li SM, et al. (2012) Transcriptome-wide survey of mouse CNS-derived cells reveals monoallelic expression within novel gene families. PLoS ONE 7(2):e31751.

12. Nag A, et al. (2013) Chromatin signature of widespread monoallelic expression. eLife 2:e01256.

13. Zwemer LM, et al. (2012) Autosomal monoallelic expression in the mouse. Genome Biol 13(2):R10.

14. Nag A, Vigneau S, Savova V, Zwemer LM, Gimelbrant AA (2015) Chromatin Signature Identifies Monoallelic Gene Expression Across Mammalian Cell Types. G3\&amp;\#58; Genes|Genomes|Genetics 5(8):1713-1720.

15. Savova V, Patsenker J, Vigneau S, Gimelbrant AA (2016) dbMAE: the database of autosomal monoallelic expression. Nucleic Acids Research 44(D1):D753-D756.

16. Pereira JP, Girard R, Chaby R, Cumano A, Vieira $P$ (2003) Monoallelic expression of the murine gene encoding Toll-like receptor 4. Nat Immunol 4(5):464470.
17. Nag A, Vigneau S, Savova V, Zwemer LM, Gimelbrant AA (2015) Chromatin Signature Identifies Monoallelic Gene Expression Across Mammalian Cell Types. G3: Genes| Genomes| Genetics 5(8):1713-1720.

18. Savova V, et al. (2016) Genes with monoallelic expression contribute disproportionately to genetic diversity in humans. Nat Genet 48(3):231-237.

19. Savova V, Vinogradova S, Pruss D, Gimelbrant AA, Weiss LA (2017) Risk alleles of genes with monoallelic expression are enriched in gain-of-function variants and depleted in loss-of-function variants for neurodevelopmental disorders. Mol Psychiatry 5:1713.

20. da Rocha ST, Gendrel A-V (2019) The influence of DNA methylation on monoallelic expression. Essays Biochem 56:100.

21. Xu J, et al. (2017) Landscape of monoallelic DNA accessibility in mouse embryonic stem cells and neural progenitor cells. Nat Genet 49(3):377-386.

22. Mendelevich A, Vinogradova S, Gupta S, et al (2020) Unexpected variability of allelic imbalance estimates from RNA sequencing. biorxiv. doi:10.1101/2020.02.13.948323.

23. Kivioja T, et al. (2011) Counting absolute numbers of molecules using unique molecular identifiers. Nat Meth 9(1):72-74.

24. Islam S, et al. (2013) Quantitative single-cell RNAseq with unique molecular identifiers. Nat Meth 11(2):163-166.

25. Rosenberg N, Baltimore D, Scher CD (1975) In vitro transformation of lymphoid cells by Abelson murine leukemia virus. Proceedings of the National Academy of Sciences 72(5): 1932-1936.

26. Jones PA, Taylor SM (1980) Cellular differentiation, cytidine analogs and DNA methylation. Cell 20(1):85-93.

27. Kamitaki N, Usher CL, McCarroll SA (2018) Using Droplet Digital PCR to Analyze Allele-Specific RNA Expression. Methods Mol Biol 1768(Chapter 23):401422.

28. Christman JK (2002) 5-Azacytidine and 5-aza-2'deoxycytidine as inhibitors of DNA methylation: mechanistic studies and their implications for cancer therapy. Oncogene 21(35):5483-5495.

29. Zhu W-G, et al. (2004) 5-aza-2'-deoxycytidine activates the p53/p21Waf1/Cip1 pathway to inhibit cell proliferation. Journal of Biological Chemistry 279(15):15161-15166.

30. Smith ZD, Meissner A (2013) DNA methylation: roles in mammalian development. Nature Publishing Group 14(3):204-220.

31. Meissner A, et al. (2008) Genome-scale DNA methylation maps of pluripotent and differentiated cells. Nature 454(7205):766-770.

32. Dunford A, et al. (2017) Tumor-suppressor genes that escape from $X$-inactivation contribute to cancer sex bias. Nat Genet 49(1):10-16. 
bioRxiv preprint doi: https://doi.org/10.1101/2020.02.20.954834; this version posted July 23, 2020. The copyright holder for this preprint (which was not certified by peer review) is the author/funder, who has granted bioRxiv a license to display the preprint in perpetuity. It is made available under aCC-BY-NC-ND 4.0 International license.

33. Damaschke NA, et al. (2017) Loss of Igf2 Gene Imprinting in Murine Prostate Promotes Widespread Neoplastic Growth. Cancer Res 77(19):5236-5247.

34. Bix M, Locksley RM (1998) Independent and Epigenetic Regulation of the Interleukin-4 Alleles in CD4+ T Cells. Science 281(5381):1352-1354.

35. Catania S, et al. (2020) Evolutionary Persistence of DNA Methylation for Millions of Years after Ancient Loss of a De Novo Methyltransferase. Cell 180(2):263277.e20.

36. Karahoca M, Momparler RL (2013) Pharmacokinetic and pharmacodynamic analysis of 5-aza-2'deoxycytidine (decitabine) in the design of its doseschedule for cancer therapy. Clin Epigenetics 5(1):3.

37. Williams BP, Pignatta D, Henikoff S, Gehring M (2015) Methylation-sensitive expression of a DNA demethylase gene serves as an epigenetic rheostat. PLoS Genet 11(3):e1005142.

38. Williams BP, Gehring M (2017) Stable transgenerational epigenetic inheritance requires a DNA methylation-sensing circuit. Nature Communications 8(1):2124.

39. Miller SA, Dykes DD, Polesky HF (1988) A simple salting out procedure for extracting DNA from human nucleated cells. Nucleic Acids Research 16(3):12151215.

40. Shen Z, et al. (2010) MPprimer: a program for reliable multiplex $\mathrm{PCR}$ primer design. BMC Bioinformatics 2010 11:1 11(1):143.

41. Qu W, Shen Z, Zhao D, Yang Y, Zhang C (2009) MFEprimer: multiple factor evaluation of the specificity of PCR primers. Bioinformatics 25(2):276-278.

42. Langmead B, Salzberg SL (2012) Fast gapped-read alignment with Bowtie 2. Nat Meth 9(4):357-359.

43. Xi Y, Li W (2009) BSMAP: whole genome bisulfite sequence MAPping program. BMC Bioinformatics 2010 11:1 10:232.

44. Tukey, JW. (1977). Exploratory data analysis. Addison-Wesely, 1 edition edn (Reading, Mass: Pearson) 